\title{
PALS probing of photopolymerization shrinkage in densely packed acrylate-type dental restorative composites
}

\author{
Olha Shpotyuk ${ }^{1, A-F}$, Adam Ingram ${ }^{2, B, C, E, F}$, Oleh Shpotyuk ${ }^{3, A, C-F}$, Andrii Miskiv ${ }^{1, B, C, E, F}$, Nina Smolar ${ }^{1, A, C, E, F}$ \\ ${ }^{1}$ Department of Orthodontics, Danylo Halytsky Lviv National Medical University, Ukraine \\ ${ }^{2}$ Department of Physics, Opole University of Technology, Poland \\ ${ }^{3}$ Faculty of Science and Technology, Jan Dlugosz University of Czestochowa, Poland \\ A - research concept and design; $\mathrm{B}$ - collection and/or assembly of data; $\mathrm{C}$ - data analysis and interpretation; \\ $\mathrm{D}$ - writing the article; $\mathrm{E}$ - critical revision of the article; $\mathrm{F}$ - final approval of the article
}

Address for correspondence

Oleh Shpotyuk

E-mail: olehshpotyuk@yahoo.com

Funding sources

None declared

Conflict of interest

None declared

Received on December 23, 2019

Reviewed on January 28, 2020

Accepted on February 23, 2020
Cite as

Shpotyuk 0, Ingram A, Shpotyuk 0, Miskiv A, Smolar N. PALS probing of photopolymerization shrinkage in densely packed acrylate-type dental restorative composites. Polim Med. 2019;49(2):49-56. doi:10.17219/pim/118394

DOI

10.17219/pim/118394

Copyright

○) 2020 by Wroclaw Medical University

This is an article distributed under the terms of the

Creative Commons Attribution 3.0 Unported (CC BY 3.0)

(https://creativecommons.org/licenses/by/3.0/)

\section{Abstract}

Background. Using positron annihilation lifetime spectroscopy (PALS), microstructural changes in commercial dental restorative composites under light-curing polymerization were identified as a modification in mixed positron/Ps trapping, where the decay of positronium (Ps; the bound state of positrons and electrons) is caused by free-volume holes mainly in the polymer matrix, and positron trapping is defined by interfacial free-volume holes in a mixed filler-polymer environment. In loosely packed composites with a filler content of $<70-75 \%$, this process was related to the conversion of Ps-to-positron trapping.

Objectives. To disclose such peculiarities in densely packed composites using the example of he commercially available acrylate-based composite ESTA-3 ${ }^{\circledR}$ (ESTA Ltd., Kiev, Ukraine), which boasts a polymerization volumetric shrinkage of only $1.5 \%$.

Material and methods. ESTA- $3^{\circledR}$ was used as a commercially available acrylate-based dental restorative composite. A fast-fast coincidence system of 230-ps resolution based on 2 photomultiplier tubes c0upled to a BaF2 detector and ORTE ${ }^{\circledR}$ electronics was used to register lifetime spectra in normal-measurement statistics. The raw PAL spectra were treated using X3-x2-CDA (coupling decomposition algorithm).

Results. The annihilation process in the densely packed dental restorative composites (DRCS), as exemplified by the commercially available acrylate-based composite ESTA- ${ }^{\circledR}$, is identified as mixed positron/Ps trapping, where o-Ps decay is caused by free-volume holes in the polymer matrix and interfacial filler-polymer regions, and free positron annihilation is defined by free-volume holes between filler particles. The most adequate model-independent estimation of the polymerization volumetric shrinkage can be done using averaged positron annihilation lifetime. A meaningful description of the transformations in Ps- and positron-trapping sites under light curing can be developed on the basis of a semiempirical model exploring $\times 3-\times 2-C D A$. There is a strong monolithization of agglomerated filler nanoparticles in these composites, caused by the photo-induced disappearing of positron traps at the cost of Ps-decaying holes.

Conclusions. Governing the polymerization void-evolution process in densely packed DRC ESTA-3 ${ }^{\circledR}$ occurs mainly in the filler sub-system as positron-to-Ps trapping conversion, which is the reason for the low corresponding volumetric shrinkage.

Key words: acrylates, positron annihilation lifetime spectroscopy, dental restorative composites, light curing, photopolymerization 


\section{Introduction}

Acrylic-type polymers filled with inorganic particles/ nanoparticles compose an important class of dental restorative composites (DRCs), which can be effectively polymerized under light curing. ${ }^{1-3}$ The monomer chains characteristic of uncured DRC are cross-linked by intramolecular bonds forming a denser polymer-composite matrix, thus producing polymerization stress or volumetric shrinkage. ${ }^{3}$

In most dental restoratives with a moderate filler content (no more than $70-80 \%$ ), which can be conditionally defined as loosely packed DRCs, the shrinkage-accompanied stress approaches 1.5-15 MPa and the volumetric shrinkage is in the range of $2-4 \%{ }^{3}$ The commercially available DRCs Charisma ${ }^{\circledR}$ (Heraeus Kulzer GmbH, Hanau, Germany) ${ }^{4}$ and Dipol ${ }^{\circledR}$ (Oksomat-AN Ltd., Kiev, Ukraine), ${ }^{5}$ can be mentioned as typical examples of such restoratives. These DRCs composed of a monomer (bisphenol A-diglycidyl dimethacrylate and triethyleneglycol dimethacrylate) modified by glass filler particles of various sizes (typically, pyrogenic silica glass $\left(\mathrm{SiO}_{2}\right)$ ) to modify weight-packing densities, possess high volumetric shrinkage under lightcuring polymerization (above $\sim 2 \%$ ).

Employing positron annihilation lifetime spectroscopy (PALS), ${ }^{6-10}$ microstructural changes under polymerization were identified as a modification in mixed positron/Ps trapping, where positronium (Ps; the bound state of positron and electron decay) is caused by freevolume holes mainly in the polymer matrix, and positron trapping is defined by interfacial free-volume holes in the filler-polymer environment. ${ }^{11-15}$ The PAL spectra of polymerized DRCs follow a multicomponent trapping model with respect to multichannel positron/Ps annihilation, with the number of physically realistic channels not being specifically defined. Most often, the PAL spectra of light-cured DRCs based on acrylate-type resins are reconstructed under partially constrained $\mathrm{x} 4$ - or $\mathrm{x} 3$-term analysis. ${ }^{16-19}$ However, the unconstrained $x 3$-term decomposition seems more suitable for experimental PAL spectra governed by mixed positron/Ps trapping. Indeed, in such case, the process of Ps-to-positron trapping conversion can be successfully parameterized employing an $\mathrm{x} 3-\mathrm{x} 2$ coupling decomposition algorithm (CDA), validated for mixed positron/Ps-related annihilation in nanocomposites. ${ }^{20-23}$

At higher concentrations of filler (above $\sim 80 \%$ ) and corresponding lower proportions of resin in the mixture, the volumetric shrinkage is typically moderated to less than $\sim 2 \%$, since the reduced amount of resin shrinks less in a given volume of composite. ${ }^{3}$ In these DRCs, the variations in particle size allow optimized particle distribution and adequate density, contributing to reduced shrinkage. This is a case of "low-shrinkage" DRCs, characterized as "densely monolithic" restoratives. This specificity in the inner composite make-up, with an increased proportion of micro- $(1-1.5 \mu \mathrm{m})$ and sub-micro-sized $(\sim 0.5 \mu \mathrm{m})$ glass or glass-ceramic particles and amorphous silica nano-sized particles $(<40 \mathrm{~nm})$ (above $80 \%)$ is thought to be associated with some changes in the underlying mechanism of free-volume modification under polymerization light-curing. In this paper, we attempt to study such peculiarities for densely packed DRCs using PALS with the commercially available acrylate-type composite ESTA- $3^{\circledR}$ (ESTA Ltd., Kiev, Ukraine), which possesses a volumetric polymerization shrinkage of only $1.5 \%{ }^{24}$

\section{Material and methods}

The specimens of ESTA- $3{ }^{\circledR}$ DRC were prepared by filling an inner volume of a disk-shaped plastic mold $(6 \mathrm{~mm}$ in diameter and $2 \mathrm{~mm}$ in thickness). The bottom surface was covered with a polyethylene slice film, which was separated from the DRC along with the outer ring around the disk prior to PALS measurements. Some of the samples were polymerized by illuminating them with a curing dental wireless LED light source (LED.T4 SEASKY, Beijing, China), emitting light in the range of $420-480 \mathrm{~nm}$ with a power density output of $\sim 900 \mathrm{~mW} / \mathrm{cm}^{2}$. Under illumination, the guide tip of the light source was maintained just above the sample surface (at a distance of $7 \mathrm{~mm}$ ) so that the light beam completely covered the sample surface. The batch of non-polymerized DRC samples was marked Dent 0 , and further batches of photopolymerized samples were marked with numbers corresponding to the light curing duration in seconds (Dent 5, Dent 20, Dent 40, and Dent 60).

The methodology of the PALS studies was identical to that of our recent research. ${ }^{11-13}$ The raw PAL spectra were registered with a fast-fast coincidence system of 230-ps resolution based on 2 Photonis XP2020/Q photomultipliers coupled to BaF2 scintillator detectors (Scionix Holland B.V., Bunnik, the Netherlands) and ORTEC ${ }^{\circledR}$ electronics (ORTEC, Oak Ridge, USA). To ensure high reliability, each spectrum involved $1 \mathrm{M}$ of elementary positron annihilation events, accumulated at a temperature of $22^{\circ} \mathrm{C}$ and a relative humidity of $35 \%, 22$ using an $\mathrm{Na}$ isotope of $\sim 50 \mathrm{kBq}$ activity as a positron source sandwiched between 2 tested samples. The PAL spectra were processed using LT v. 9.0 program, ${ }^{25}$ stabilizing the average positron lifetime, $\tau_{a v}^{\Sigma}$ as:

$$
\tau_{a v}^{\Sigma}=\sum_{i} I_{i} \tau_{i}
$$

where $\tau_{i}$ and $I_{i}$ denote positron lifetime and the intensity of the corresponding fitting components (the accuracy in lifetime $\tau_{i}$ and intensity $I_{i}$ was $5 \pm 0.005$ ns and $\pm 0.5 \%$, respectively).

The best fit of the collected PAL spectra for polymeric materials is achieved via mixed channels of trapping, which occurs from defect-related positron traps 
and bound positron-electron states, i.e., positronium. This can be solved through the multi-component fitting of the PAL spectrum with 3 or 4 single exponentials under unconstrained (free-fitting components) or constrained (used most often for some fixed fitting parameters, such as the shortest positron lifetime, maintained close to $0.125 \mathrm{~ns}$ ) decomposition procedures and normalized component intensities $(i=3$ or 4$)$

$$
\sum_{i} I_{i}=1
$$

Thus, the fitting covers realistic channels caused by positrons, which annihilate from delocalized states in defect-free bulk, and those trapped from spatially-extended free-volume defects (positron trapping) and Ps states through "pick-up" annihilation with an electron from the surrounding material (Ps decaying).

Ignoring the contribution from Ps decay, these spectra can be parameterized in terms of the canonical two-state simple trapping model (STM) with one kind of defect, parameters of defect-free lifetime $t_{b}$, the trapping rate in defects $k_{d}$, and the percentage of trapped positrons $h .^{6,8,10}$ The other channel is caused by annihilation from the Ps state as free particles or those interacting with electrons from the environment. ${ }^{7,9,10}$ In a ground state, Ps exists as para-Ps (antiparallel spins), decaying intrinsically with 2 g-quanta and a 0.125 -ns lifetime in a vacuum, and as ortho-Ps (parallel spins), decaying with 3 g-quanta and a 142-ns lifetime. In matter, since positron wave function overlaps with electron wave function outside, annihilation with such electrons having an antiparallel spin decreases their lifetime to $0.5-10 \mathrm{~ns}$, resulting in 2 gamma-rays "pick-off" annihilation. Ps localized in free-volume holes provides an indication on their mean radii $R$ in terms of $\tau_{3}$ lifetime ( $I_{3}$ value correlates with the density of Ps sites) with respect to the Tao-Eldrup equation:

$\tau_{3}=0.5 \cdot\left[1-\frac{R}{R+\Delta R}+\frac{1}{2 \pi} \cdot \sin \left(\frac{2 \pi R}{R+\Delta R}\right)\right]^{-1}$

where $\Delta R=0.166 \mathrm{~nm}$ is the fitted empirical layer thickness. ${ }^{7}$

By fitting Equation 2 with the measured $\tau_{3}$ values, radius $R_{3}$ and the free volumes $V_{f}$ can be determined, making it possible to calculate the fractional free volume $f_{v}{ }^{3}$ using the empirical constant, $C=0.0018 \AA^{-3}$.

$$
f_{v}^{3}=C \cdot I_{3} \cdot V_{f}
$$

The PAL spectra in polymer-filler DRCs are known to be composed of mixed positron/Ps trapping channels. ${ }^{11-13}$ If Ps-decay and positron-trapping sites are interconnected so that no changes occur in other channels, we can treat these spectra with $x 3-x 2-C D A .{ }^{20-23}$ Within this approach, the experimental $\mathrm{x} 3$-term PAL spectra are transformed into the generalized $\mathrm{x} 2$-term form for host (initial or nonpolymerized) and modified (polymerized) DRCs. At this stage, we can simply resolve an additional second component $\left(t_{i n t}, I_{\text {int }}\right)$ for a polymerized DRC with defect-related lifetime $t_{i n t}$ and intensity $I_{i n t}$, as well as compensate component with the input in the first channel $\left(t_{n}, I_{n}\right)$, assuming a reasonable condition of full inter-channel equilibrium. The interchangeable Ps/positron traps in the polymerized DRC can be parameterized accepting $\left(t_{n}, I_{n}\right)$ and $\left(t_{\text {int }}, I_{\text {int }}\right)$ as the first and second components, respectively, of the $\mathrm{x} 2$-term decomposed spectrum for some hypothetical medium which obeys the formalism of canonical twostate STM. ${ }^{6,8,10}$ The defect-related lifetime $t_{\text {int }}$ in this model reflects the appearing/disappearing traps depending on the positive/negative sign of both $I_{n}$ and $I_{\text {int }}$ intensities. ${ }^{20}$

In case of a stronger input from Ps decaying in the $\mathrm{x} 3$-term PAL spectrum, positron trapping can be defined in terms of the same STM, assuming 2 additional contributions from trapped positrons (the positron trapping component) and o-Ps (the o-Ps trapping component). ${ }^{12,26}$ This model with 2 additional positron-trapping defects with $\kappa_{d 1}$ and $\kappa_{d 2}$ annihilation rates, defined as

$$
\kappa_{d 1}=I_{2}\left(\frac{1}{\tau_{1}}-\frac{1}{\tau_{2}}\right)
$$

and

$$
\kappa_{d 2}=I_{3}\left(\frac{1}{\tau_{1}}-\frac{1}{\tau_{3}}\right)
$$

allows a more correct estimation of bulk lifetime $t_{b}$ related to annihilation from Bloch states ${ }^{20}$ :

$$
\tau_{b}=\left(\frac{I_{1}}{\tau_{1}}+\frac{I_{2}}{\tau_{2}}+\frac{I_{3}}{\tau_{3}}\right)^{-1}
$$

\section{Results and discussion}

The PAL spectra of ESTA- $3^{\circledR}$ DRC in the initial nonpolymerized state (Dent 0 ) and after respective 60 -second light exposure (Dent 60 sample) subjected to free $\mathrm{x} 3$-term decomposition are depicted in Fig. $1 \mathrm{a}$ and $1 \mathrm{~b}$, respectively. The narrow-restricted statistical scatter of variance tightly grouped around the horizontal axis at the bottom of Fig. 1 demonstrates that PALS measurements are adequately described within this decomposition procedure. The respective best-fit parameters, positron-trapping and Ps-decay modes are presented in Tables 1 and 2 .

With respect to these data, the annihilation in all ESTA- $3^{\circledR}$ DRC samples can be identified as mixed positron/Ps trapping, where the $3^{\text {rd }}$ Ps decay component originates from free-volume holes in the polymer matrix, 

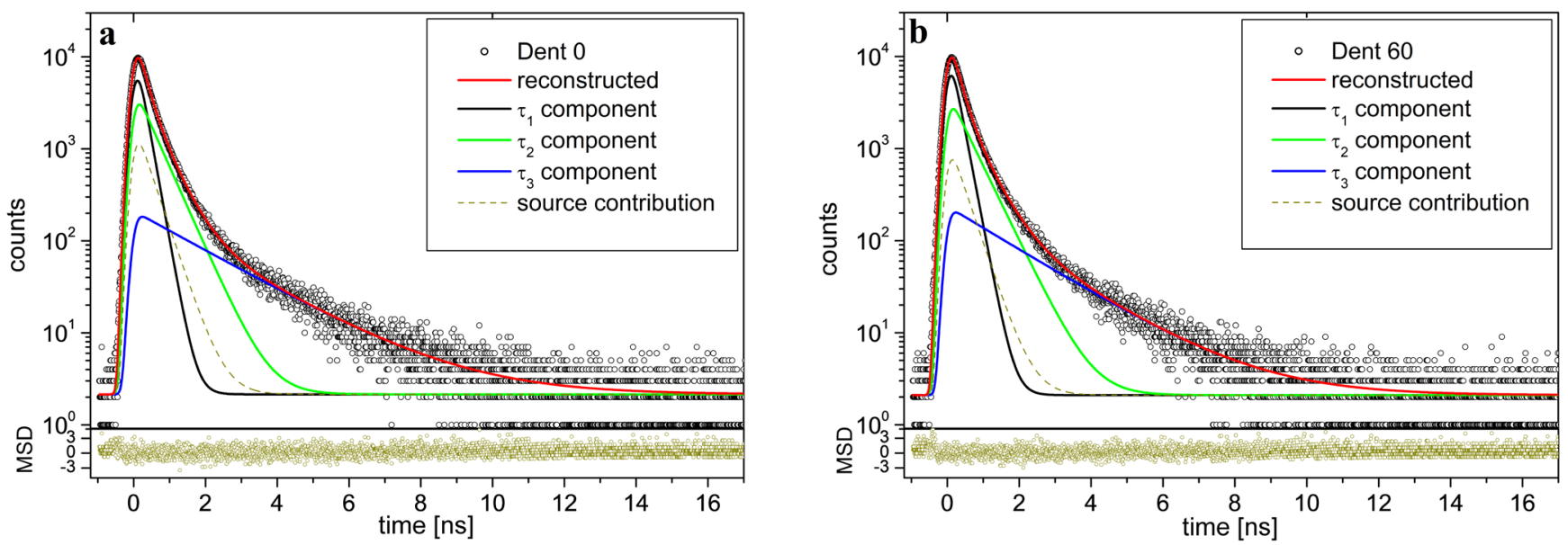

Fig. 1. Positron annihilation lifetime (PAL) spectra of non-polymerized dental restorative composite (DRC) Dent 0 (a) and polymerized DRC Dent 60 (b), reconstructed from unconstrained $\times 3$-fitting at the general background of source contribution; the bottom insets show the statistical scatter of variance - mean square deviation (MSD).

Table 1. The best-fit PAL spectra parameters for ESTA- $3^{\circledR}$ DRC determined with an unconstrained x3-term decomposition procedure

\begin{tabular}{|c|c|c|c|c|c|c|c|}
\hline $\begin{array}{c}\text { DRC exposure } \\
{[\mathrm{s}]}\end{array}$ & [FIT-1] & $\begin{array}{c}\tau_{1} \\
{[\mathrm{~ns}]}\end{array}$ & $\begin{array}{c}\tau_{2} \\
{[n s]}\end{array}$ & $\begin{array}{c}\tau_{3} \\
{[\mathrm{~ns}]}\end{array}$ & $\begin{array}{c}I_{2} \\
{[\text { a.u. }]}\end{array}$ & $\begin{array}{c}l_{3} \\
{[\text { a.u.] }}\end{array}$ & $\begin{array}{l}\tau_{\text {avg }} \\
{[n s]}\end{array}$ \\
\hline Dent 0 & 0.01 & 0.202 & 0.501 & 2.030 & 0.430 & 0.075 & 0.468 \\
\hline Dent 5 & 0.07 & 0.204 & 0.512 & 1.924 & 0.410 & 0.077 & 0.465 \\
\hline Dent 20 & 0.04 & 0.208 & 0.518 & 1.813 & 0.410 & 0.080 & 0.462 \\
\hline Dent 40 & 0.06 & 0.208 & 0.521 & 1.779 & 0.410 & 0.081 & 0.463 \\
\hline Dent 60 & 0.01 & 0.211 & 0.529 & 1.768 & 0.390 & 0.080 & 0.460 \\
\hline
\end{tabular}

Table 2. The best-fit PAL spectra parameters for ESTA- $3^{\circledR}$ DRC determined with an unconstrained $x$-term decomposition procedure

\begin{tabular}{|c|c|c|c|c|c|c|c|c|c|}
\hline \multirow{2}{*}{$\begin{array}{c}\text { DRC exposure } \\
{[\mathrm{s}]}\end{array}$} & \multicolumn{6}{|c|}{$\mathrm{e}^{+}$-trapping modes } & \multicolumn{3}{|c|}{ Ps-decaying modes } \\
\hline & $\begin{array}{l}\tau_{\text {avgr }}^{t r} \\
{[\mathrm{~ns}]}\end{array}$ & $\begin{array}{c}\tau_{b} \\
{[n s]}\end{array}$ & $\begin{array}{c}K_{d} \\
{\left[n s^{-1}\right]}\end{array}$ & $\begin{array}{c}\tau_{2}-\tau_{b} \\
{[\mathrm{~ns}]}\end{array}$ & $\begin{array}{l}T_{2} / \tau_{b} \\
{[\text { a.u.] }}\end{array}$ & $\begin{array}{c}n \\
{[\text { a.u. }]}\end{array}$ & $\begin{array}{c}R_{3} \\
{[\mathrm{~nm}]}\end{array}$ & $\begin{array}{c}V_{f} \\
{\left[10^{-3} \mathrm{~nm}^{3}\right]}\end{array}$ & $\begin{array}{l}f_{v}^{3} \\
{[\%]}\end{array}$ \\
\hline Dent 0 & 0.340 & 0.279 & 1.36 & 0.222 & 1.80 & 0.27 & 0.291 & 103 & 1.40 \\
\hline Dent 5 & 0.342 & 0.280 & 1.32 & 0.232 & 1.83 & 0.27 & 0.280 & 92 & 1.28 \\
\hline Dent 20 & 0.345 & 0.283 & 1.28 & 0.235 & 1.83 & 0.27 & 0.270 & 82 & 1.18 \\
\hline Dent 40 & 0.346 & 0.283 & 1.27 & 0.238 & 1.84 & 0.26 & 0.267 & 80 & 1.16 \\
\hline Dent 60 & 0.347 & 0.284 & 1.21 & 0.245 & 1.86 & 0.26 & 0.266 & 80 & 1.12 \\
\hline
\end{tabular}

and the $2^{\text {nd }}$ component is defined by positron traps located mainly in the filler. ${ }^{12}$ A comparative presentation of the $\mathrm{x} 3$-term-decomposed PAL spectra for the non-polymerized Dent 0 and the polymerized Dent 60 samples in Fig. 2 speaks in favor of a decrease in average positron lifetime $t_{\text {avg }}$ under polymerization due to a shorter "tail" in the histogram of positron annihilation events connected with Ps decay. With longer photopolymerization, the $\tau_{3}$ lifetime shows a distinct 13\% decrease (from 2.030 to $1.788 \mathrm{~ns}$ under 60 -second exposure), while $I_{3}$ intensity clearly grows by $7 \%$ (Table 1). The radii of Ps-decay holes in these DRCs estimated in a spherical approximation using Equation 2 show a monotonically decreasing trend from $0.291 \mathrm{~nm}$ (for Dent 0) to $0.266 \mathrm{~nm}$ (for Dent 60). The sharp decrease in $\tau_{3}$ results in systematic decaying in the fractional free volumes $f_{v}{ }^{3}$ was calculated with Equation 4.
In contrast, the changes observed in the $2^{\text {nd }}$ component are opposite to light-curing duration, the $\tau_{2}$ lifetime demonstrating an increasing trend (from $0.501 \mathrm{~ns}$ for Dent 0 to $0.529 \mathrm{~ns}$ for Dent 60 ) and the respective $I_{2}$ intensity decreasing (Table 1). Under essential input from Ps decay, as in the case of ESTA- $3^{\circledR}$ DRC, parameterization of this channel in terms of a two-state STM is meaningless. Nevertheless, given the obvious reverse trend in both intensities $I_{3}$ and $I_{1}$ with respect to $I_{2}$ (Table 1), these changes in the PAL spectra with increased light curing duration can be attributed to preferential modification in the probability of Ps formation, ${ }^{27}$ thus favoring positron-to-Ps trapping conversion in polymerized ESTA- $3{ }^{\circledR}$ DRC described by x3-x2-CDA. ${ }^{20-23}$

The more realistic values of defect-free bulk positron lifetime $t_{b}$ related to positron annihilation from Bloch states can be extracted from STM assuming additional positron-trapping inputs from both trapped positrons 


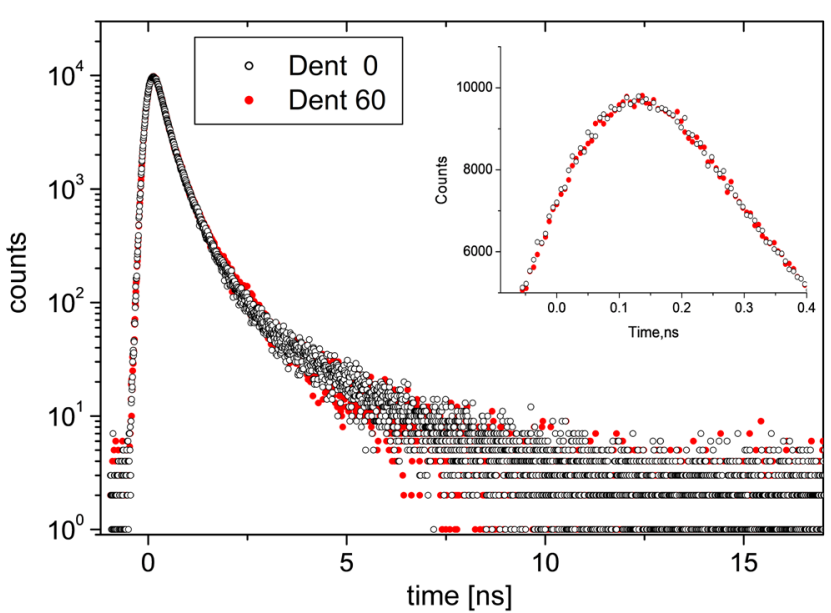

Fig. 2. PAL spectra of non-polymerized DRC Dent 0 (open circles) compared with the polymerized DRC Dent 60 (full red circles), reconstructed from unconstrained $\times 3$-term decomposition (the inset shows a comparison of annihilation events accumulated in a peak)

and decayed o-Ps states (with the positron lifetimes $\tau_{2}$ and $\tau_{3}$ given in Table 1). This $t_{b}$ value, estimated by Equation 7 , approaches $0.30 \mathrm{~ns}$ (Table 4 ) and is nearly $15 \%$ longer than that extracted from the constraint-free $\mathrm{x} 3$-term decomposition (Table 2). It is clear from Table 3 that light-curing polymerization leads to a decrease in the rate of "purely positron" trapping $\mathrm{k}_{\mathrm{d} 1}$ (by nearly $16 \%$ ) compared to the trapping rate of "o-Ps decaying" channel $k_{d 2}$, which remains essentially the same.

The PAL spectra of ESTA- $3^{\circledR}$ DRC in the initial state (Dent 0 ) and after $60 \mathrm{~s}$ of exposure (Dent 60) subjected to partially constrained $\mathrm{x} 4$-term decomposition and as-
Table 4. PAL trapping modes for ESTA $-3^{\circledR}$ DRC within an unconstrained x3-term decomposition, assuming 2 additional positron-trapping defect states

\begin{tabular}{|c|c|c|c|c|}
$\begin{array}{c}\text { DRC exposure } \\
{[\mathrm{s}]}\end{array}$ & $\begin{array}{c}\tau_{\text {avg }}{ }^{\mathrm{n}} \\
{[\mathrm{ns}]}\end{array}$ & $\begin{array}{c}\tau_{b} \\
{[\mathrm{~ns}]}\end{array}$ & $\begin{array}{c}K_{d 1} \\
{\left[\mathrm{~ns}^{-1}\right]}\end{array}$ & $\begin{array}{c}K_{d 2} \\
{\left[\mathrm{~ns}^{-1}\right]}\end{array}$ \\
\hline Dent 0 & 0.468 & 0.297 & 1.27 & 0.33 \\
Dent 60 & 0.460 & 0.304 & 1.11 & 0.33 \\
\hline
\end{tabular}

suming $\tau_{1}=0.125 \mathrm{~ns}$ (i.e., fixed at p-Ps self-annihilation lifetime) are depicted in Fig. 3a and 3b, respectively; the best-fit parameters, positron-trapping and Ps-decay modes under this decomposition procedure are presented in Table 4. Such an analysis was shown to be reasonable for polymer-matrix composites, where the basic polymer shows bifurcation in o-Ps lifetimes, as in, for example, some semicrystalline polymers. ${ }^{28,29}$ In this case, the $4^{\text {th }}$ component is attributed to o-Ps pick-off annihilation in holes of amorphous structures $\left(\tau_{4} \approx 2-4 \mathrm{~ns}\right)$, and the $3^{\text {rd }}$ component is ascribed to o-Ps pick-off annihilation in interstitial free-volume voids of crystalline phase ( $\left.\tau_{3} \approx 1 \mathrm{~ns}\right)$.

Still, this is not the current case, since the polymer matrix of ESTA- ${ }^{\circledR}$ DRC based on bisphenol A polycarbonates is solely characterizedbyx3-term PAL spectra, where the only long-lived component comes from o-Ps decay. ${ }^{30-32}$ Simple physical mixing in the $2^{\text {nd }}$ component of the $x 4$-term-decomposed PAL spectra originating from interfacial holes and other free-volume defects in a solid/polymer phase cannot be excluded to separate realistic physical channels. Therefore, this analysis provides an invalid parameterization of the $2^{\text {nd }}$ component as an artifact of inadequately

Table 3. PAL spectra fitting parameters for ESTA- $3^{\circledR}$ DRC within a partially constrained $x$ 4-term decomposition $\left(\tau_{1}=0.125 \mathrm{~ns}\right)$

\begin{tabular}{|c|c|c|c|c|c|c|c|c|}
\hline \multirow{2}{*}{$\begin{array}{c}\text { DRC exposure } \\
{[s]}\end{array}$} & \multirow[b]{2}{*}{ [FIT-1] } & \multicolumn{6}{|c|}{ PAL spectra fitting parameters } & \multirow[b]{2}{*}{$\begin{array}{c}\tau_{\text {avgr }} \\
\mathrm{ns}\end{array}$} \\
\hline & & $\begin{array}{c}\tau_{2} \\
{[\mathrm{~ns}]}\end{array}$ & $\begin{array}{c}\tau_{3} \\
{[\mathrm{~ns}]}\end{array}$ & $\begin{array}{l}\tau_{4,} \\
\text { [ns }\end{array}$ & $\begin{array}{l}I_{2,} \\
\text { a.u. }\end{array}$ & $\begin{array}{l}I_{3,} \\
\text { a.u. }\end{array}$ & $\begin{array}{l}I_{4}, \\
\text { a.u. }\end{array}$ & \\
\hline Dent 0 & 0.01 & 0.244 & 0.539 & 2.070 & 0.45 & 0.356 & 0.072 & 0.465 \\
\hline Dent 60 & 0.01 & 0.227 & 0.547 & 1.788 & 0.51 & 0.363 & 0.077 & 0.459 \\
\hline
\end{tabular}
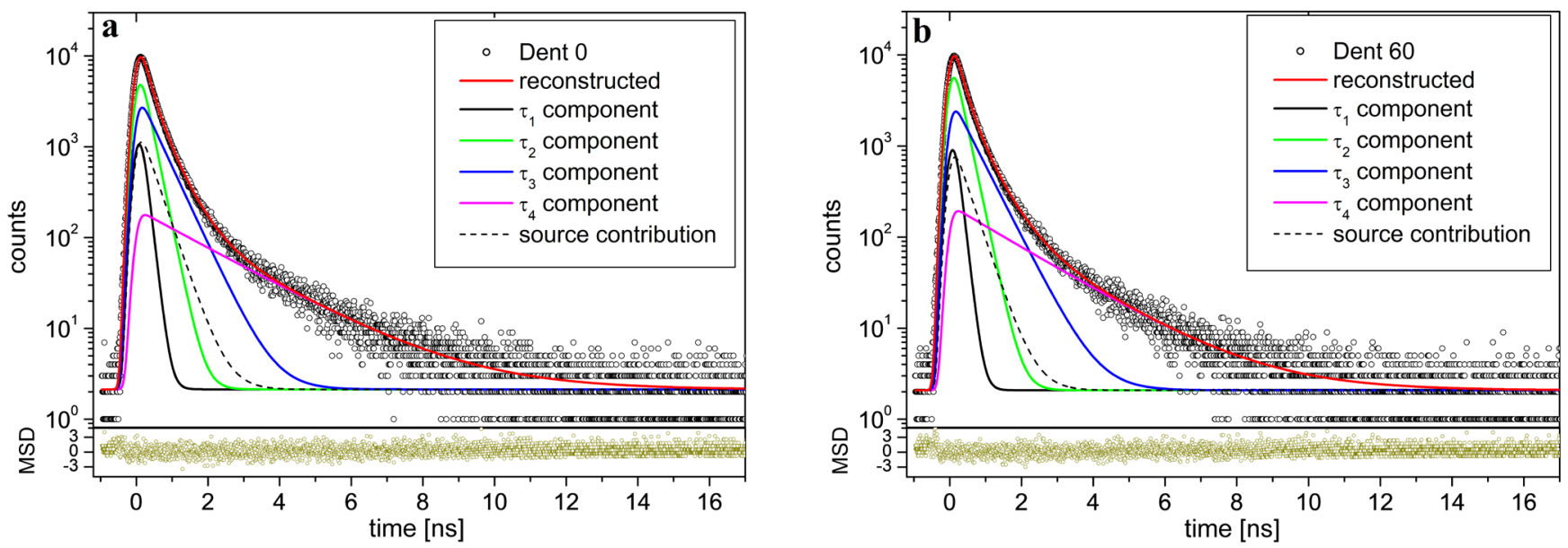

Fig. 3. PAL spectra of non-polymerized DRC Dent 0 (a) and polymerized DRC Dent 60 (b), reconstructed from partially constrained x4-fitting under fixed $\tau_{1}=0.125 \mathrm{~ns}$ at the general background of source contribution; the bottom insets show the statistical scatter of variance - mean square deviation (MSD) 
fitting $x 4$. In case of multiple o-Ps decays of the same origin, this component can be easily replaced by apparent lifetime, which is a mean value averaged over all o-Ps components with corresponding intensities. ${ }^{33}$ It can clearly be seen from Table 4 that changes in the $3^{\text {rd }}$ component can indeed be ignored, thus transforming the partially constrained (with $\tau_{1}=0.125 \mathrm{ns)}$ x4-term-decomposed PAL spectra into free $\mathrm{x} 3$-term-decomposed ones (Table 1).

It is known that when free-volume voids are mutually inter-transformed under trapping conversion, the x3-term-decomposed PAL spectra can be treated using the $\mathrm{x} 3-\mathrm{x} 2-\mathrm{CDA}$ for uncured DRCs with a light-cured one (in this case, both $I_{n}$ and $I_{i n t}$ are positive); the results of such treatment for the current DRC are presented in Table 5. This analysis shows that positron trapping sites like triple junctions, mainly between agglomerated filler particles with character-defect-specific lifetimes of $\sim 0.34-0.36 \mathrm{~ns}$, disappear under light curing in favor of o-Ps-decaying holes with lifetimes of $\sim 1.7-1.8 \mathrm{~ns}$ and corresponding to decreased free volumes in the polymer matrix. These disappearing positron traps are like triple junctions between agglomerated filler particles, located in filler environment, as it follows from the low $t_{B}$ in Table 5 - with values typical of nanosized glassy particles of silica or zirconia. ${ }^{34}$ In other words, agglomerated filler particles/nanoparticles are strongly monolithized under light-curing polymerization in these DRCs, caused by photo-induced cross-linking of structural chains in the polymer matrix.

In Table 5, x3-x2-CDA data calculated for Dent 40 in comparison with Dent 60 is also presented, allowing a comparison of the initial (0-20 s) and final (40-60 s) stages of light-curing polymerization. It is noteworthy that these stages differ only by defect-specific $t_{\text {int }}$ and defect-free $t_{B}$ positron lifetimes, meaning that under initial exposure the larger free-volume positron traps (with 0.358-ns lifetimes) disappear in looser filler packing (because $t_{B}=0.208 \mathrm{~ns}$ ), while the finest free-volume voids (with 0.312-ns lifetimes) disappear under final exposure in a denser filler environment (because $t_{B}=0.182 \mathrm{~ns}$ ). This feature explains the lower degree of volumetric shrinkage under polymerization in the densely monolithic ESTA- $3^{\circledR}$ DRC as compared with other available loosely monolithic DRCs, particularly, the previously studied DRCs Charis$\mathrm{ma}^{\circledR}$ and Dipol ${ }^{\circledR} \cdot{ }^{11-13}$
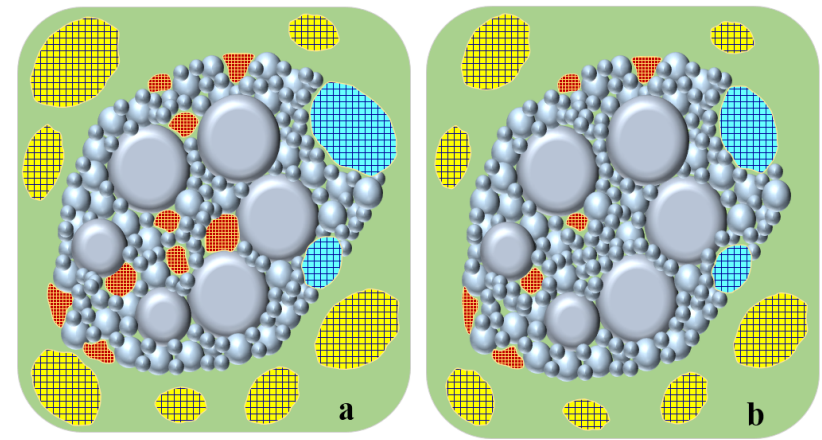

Fig. 4. Schematic view of the fragmentation of free-volume Ps and positron traps in light-cured DRC: a) microstructure fragment of agglomerated filler particles (grey) in a non-polymerized DRC matrix (green), containing an o-Ps-trapping void located in the interfacial filler-polymer region (bluecross-dashed line), o-Ps-trapping holes mainly in the polymer matrix (yellow-cross-dashed line) and positron trapping sites in the filler matrix (red-cross-dashed line); b) the same agglomerate of filler particles in a polymerized DRC matrix (an o-Ps-trapping void in the interfacial fillerpolymer region disappears, giving rise to more contracted o-Ps-trapping voids in the surrounding polymer matrix and reduced trapping sites within the agglomerated filler particles)

The results described above lead to a complete protocol of light-curing polymerization for ESTA-3 DRC (Fig. 4). In the initial non-polymerized state (Fig. 4a), these densely packed DRCs are filled in positron traps located preferentially in the filler sub-system (Fig. 4a, red-cross-dashed lines), as well as Ps-decaying voids placed in the interfacial filler-polymer region (blue-cross-dashed lines) and the polymer matrix (yellow-cross-dashed lines). Lightcuring polymerization causes cross-linking of the latter, thus resulting in the fragmentation of the Ps decay sites in the polymer matrix (Fig. 4b, yellow-cross-dashed lines). The o-Ps-trapping voids in the interfacial filler-polymer region (blue-cross-dashed lines, Fig. 4b) are also reduced in size, and some of them disappear, giving rise to more tight contact between the outermost polymer surface, completely covering agglomerated filler particles. Under further progressive polymerization, strong contraction stress suppresses the agglomerate of filler particles, reducing the size and number of intrinsic free-volume positron traps (red-cross-dashed lines in Fig. 4b). Thus, the most substantial changes in free volume mainly take place in the filler sub-system, which is a reason for the low value of the corresponding volumetric shrinkage of this DRC.

Table 5. Positron-trapping modes for non-polymerized Dent 0 sample, calculated with respect to light-cured ESTA-3 ${ }^{\circledR}$ DRC samples employing x3-x2 coupling decomposition algorithm (CDA)

\begin{tabular}{|c|c|c|c|c|c|c|c|c|c|}
\hline \multirow[b]{2}{*}{ DRC system } & \multicolumn{2}{|c|}{ I component } & \multicolumn{2}{|c|}{ II component } & \multicolumn{5}{|c|}{$\mathrm{e}^{+}$-trapping modes } \\
\hline & $\begin{array}{c}\tau_{n} \\
{[n s]}\end{array}$ & $\begin{array}{c}I_{n} \\
\text { [a.u.] }\end{array}$ & $\begin{array}{c}\tau_{\text {int }} \\
\text { [ns] }\end{array}$ & $\begin{array}{c}l_{\text {int }} \\
\text { [a.u.] }\end{array}$ & $\begin{array}{c}\tau_{a v} \\
{[\mathrm{~ns}]}\end{array}$ & $\begin{array}{c}\tau_{B} \\
{[\mathrm{~ns}]}\end{array}$ & $\begin{array}{c}K_{\mathrm{d}} \\
{\left[\mathrm{ns}^{-1}\right]}\end{array}$ & $\begin{array}{c}\tau_{\text {int }}-\tau_{B} \\
\text { [ns] }\end{array}$ & $\begin{array}{l}\tau_{\text {int }} / \tau_{B} \\
\text { [a.u.] }\end{array}$ \\
\hline Dent 0 - Dent 5 & 0.173 & 0.029 & 0.358 & 0.031 & 0.268 & 0.236 & 1.539 & 0.122 & 1.52 \\
\hline Dent 0 - Dent 20 & 0.150 & 0.049 & 0.358 & 0.046 & 0.250 & 0.208 & 1.859 & 0.150 & 1.72 \\
\hline Dent 0 - Dent 40 & 0.153 & 0.052 & 0.350 & 0.050 & 0.250 & 0.211 & 1.805 & 0.139 & 1.66 \\
\hline Dent 0 - Dent 60 & 0.149 & 0.067 & 0.342 & 0.064 & 0.243 & 0.205 & 1.862 & 0.137 & 1.67 \\
\hline Dent 40 - Dent 60 & 0.133 & 0.017 & 0.312 & 0.015 & 0.217 & 0.182 & 2.027 & 0.130 & 1.71 \\
\hline
\end{tabular}


Finally, under the contribution of mixed positron/Ps trapping channels in the overall annihilation process in composites, one of the best estimations of volumetric polymerization shrinkage, whichever the spectra-reconstruction algorithms, is related to the average lifetime defined as mass center of the registered PAL spectrum defined by Equation 1. As follows from Table 1, light curing results in a decrease in $t_{a v g}$, from $0.468 \mathrm{~ns}$ for non-polymerized DRCs to 0.460 ns for completely polymerized DRCs. The relative change (1.7\%) happens to be surprisingly close to the polymerization volumetric shrinkage of $1.5 \%$, which is characteristic of ESTA- $3{ }^{\circledR} .24$

\section{Conclusions}

Some peculiarities of volumetric shrinkage under photopolymerization are studied using PALS in a densely packed DRC - the commercially available acrylate-based composite ESTA- $3^{\circledR}$. The PAL spectra are reconstructed from unconstrained $\mathrm{x} 3$-term and partially constrained $\mathrm{x} 4$-term fitting routes assuming the shortest lifetime fixed at a theoretical value of intrinsic para-Ps self-annihilation (0.125 ns), and a simple trapping model assuming additional inputs from trapped positrons and decayed o-Ps states.

With respect to the data obtained, the annihilation in these DRCs is identified as mixed positron/Ps trapping, where the contribution from o-Ps decay is caused by free-volume holes in the polymer matrix and interfacial filler-polymer voids, and the free positron annihilation is defined by free-volume holes between the filler particles. The most adequate model-independent estimation of photopolymerization volumetric shrinkage is achieved using the average positron annihilation lifetime. A meaningful description of the transformations between Ps- and positron-trapping sites under light curing was developed on the basis of a semi-empirical model exploring the $\mathrm{x} 3-\mathrm{x} 2-\mathrm{CDA}$. Under polymerization, the most substantial changes in free volume occur mainly in the filler sub-system, which is the reason for the low value of corresponding volumetric shrinkage of this DRC.

\section{ORCID iDs}

Olha Shpotyuk (1) https://orcid.org/0000-0002-9572-1817 Adam Ingram (1) https://orcid.org/0000-0002-8424-2298 Oleh Shpotyuk (1) https://orcid.org/0000-0002-2455-1857 Andrii Miskiv (1) https://orcid.org/0000-0001-7762-5867 Nina Smolar (D) https://orcid.org/0000-0001-8593-5435

\section{References}

1. Bland MH, Peppas NA. Photopolymerized multifunctional (meth) acrylates as model polymers for dental application. Biomaterials. 1996;17:1109-1114. doi:10.1016/0142-9612(96)85912-6

2. Cramer NB, Stansbury JW, Bowman CN. Recent advantages and developments in composite dental restorative materials. J Dent Res. 2011;90:402-416. doi:10.1177/0022034510381263

3. Miletic V (ed). Dental Composite Materials for Direct Restorations. Cham Switzerland: Springer Nature; 2018:319. doi:10.1007/978-3-319-60961-4
4. Charisma ${ }^{\circledR}$. Scientific Information. Heraeus Kulzer GmbH, Hanau, Germany. https://www.pantelides-dental.gr/userfiles/files/CharismaScientificlnformation.pdf. Accessed on April 24, 2020.

5. Dipol ${ }^{\circledR}$. Composite Universal. Instruction on using Dipol materials. Oksomat-AN, Ukraine Dental Products, 2016;6-7. www.oksomatan.com. Accessed on April 24, 2020.

6. Krause-Rehberg R, Leipner HS. Positron Annihilation in Semiconductors: Defect Studies. Heidelberg, Germany: Springer 1999:383.

7. Jean YC. Positron annihilation spectroscopy for chemical analysis: A novel probe for microstructural analysis of polymers. Microchem J. 1990;42:72-102. doi:10.1016/0026-265X(90)90027-3

8. Shpotyuk O, Filipecki J. Free Volume in Vitreous Chalcogenide Semiconductors: Possibilities of Positron Annihilation Lifetime Study. Czestochowa, Poland: WSP; 2003:114.

9. Jean YC, Van Horn JD, Hung WS, Lee KR. Perspective of positron annihilation spectroscopy in polymers. Macromolecules. 2013;46:7133-7145. doi:10.1021/ma401309x

10. Tuomisto F, Makkonen I. Defect identification in semiconductors with positron annihilation: Experiment and theory. Rev Mod Phys. 2013;85:1583-1631. doi:10.1103/RevModPhys.85.1583

11. Shpotyuk O, Ingram A, Shpotyuk O. Free volume structure of acrylictype dental nanocomposites tested with annihilating positrons. Nanoscale Res Lett. 2016;11:528-1-528-6. doi:10.1186/s11671-016-1751-8

12. Shpotyuk O, Ingram A, Shpotyuk O, Bezvushko E. Light-cured dimethacrylate dental restorative composites under a prism of annihilating positrons. Polim Med. 2017;47:91-100. doi:10.17219/pim/81450

13. Shpotyuk O, Adamiak S, Bezvushko $E$, et al. Light-curing volumetric shrinkage in dimethacrylate-based dental composites by nanoindentation and PAL study. Nanoscale Res Lett. 2017;12:75-1-75-6. doi: 10.1186/s11671-017-1845-y

14. Chakraverty S, Mitra S, Mandal K, Nambissan PMG, Chattopadhyay S. Positron annihilation studies of some anomalous features of $\mathrm{NiFe}_{2} \mathrm{O}_{4}$ nanocrystals grown in $\mathrm{SiO}_{2}$. Phys Rev B. 2005;71:024115-1-8. doi:10.1103/PhysRevB.71.024115

15. Mitra S, Mandal K, Sinha S, Nambissan PMG, Kumar S. Size and temperature dependent cationic redistribution in $\mathrm{NiFe}_{2} \mathrm{O}_{4}\left(\mathrm{SiO}_{2}\right)$ nanocomposites: Positron annihilation and Mössbauer studies. J Phys D: Appl Phys. 2006;39:4228-4235. doi:10.1088/0022-3727/39/19/016

16. Kleczewska J, Bieliński DM, Dryzek E, Piatkowska A. Application of positron annihilation lifetime spectroscopy in studies of dental composites based on dimethacrylate resins. In: Pielichowski K, ed. Modern Polymeric Materials For Environmental Application, 4(1). Krakow, Poland: TEZA; 2010:143-150.

17. Kleczewska J, Bielinski DM, Ranganathan N, Sokolowski J. Characterization of light-cured dental composites. In: Ranganathan N, ed. Materials Characterization. Modern Methods and Applications. Boca Raton, USA: CRC Press; 2016:117-148.

18. Shirazinia M, Mehmandoost-Khajen-Dad A, Dehghani V, Mehmandoost-Khajen-Dad J, Khaghani M. The effect of curing light intensity on free volume size in some dental composites. Polim Med. 2016;46:129-133. doi:10.17219/pim/68647

19. Svajdlenkova $H$, Sausa $O$, Peer G, Gorsche $C$. In situ investigation of the kinetics and microstructure during photopolymerization by positron annihilation technique and NIR-photorheology. RSC Adv. 2018;8:37085-1-7. doi:10.1039/C8RA07578F

20. Shpotyuk O, Filipecki J, Ingram A, et al. Positronics of subnanometer atomistic imperfections in solids as a high-informative structure characterization tool. Nanoscale Res Lett. 2015;10:77-1-5. doi:10.1186/s11671-015-0764-z

21. Shpotyuk O, Ingram A, Filipecki J, Bujňáková Z, Baláž P. Positron annihilation lifetime study of atomic imperfections in nanostructurized solids: On the parameterized trapping in wet-milled arsenic sulfides $\mathrm{As}_{4} \mathrm{~S}_{4}$. Phys Stat Solidi B. 2016;253:1054-1059. doi:10.1002/pssb.201552560

22. Shpotyuk Ya, Cebulski J, Ingram A, Shpotyuk O. Mathematical modelling of elementary trapping-reduction processes in positron annihilation lifetime spectroscopy: Methodology of Ps-to-positron trapping conversion. J Phys (Conf Ser). 2017;936:012049-1-012049-4. doi:10.1088/1742-6596/936/1/012049

23. Shpotyuk O, Ingram A, Shpotyuk Y. Free-volume characterization of nanostructurized substances by positron annihilation lifetime spectroscopy. Nucl Instr Meth Phys Res B. 2018;416:102-109. doi:10.1016/j.nimb.2017.12.012 
24. $Э$ СТA- $3^{\circledR}$. Dental photocured material for tooth filling. ЭСТА-3 microhybrid. Instruction on using. Ukraine, Kiev (2016). http://www.esta-dental.kiev.ua/downloads/download/esta-3. pdf. Accessed December 23, 2019.

25. Kansy J. Microcomputer program for analysis of positron annihilation lifetime spectra. Nucl Instr Meth Phys Res A. 1996;374:235-244. doi:10.1016/0168-9002(96)00075-7

26. Liu M, Kitai AH, Mascher P. Point defects and luminescence centers in zinc oxide and zinc oxide doped with manganese. J Luminescence. 1992;54:35-42. doi:10.1016/0022-2313(92)90047-D

27. Vijay YK, Wate S, Awasthi DK, Das D, Ghughre S. Ion induced effects in polymers. Indian J Eng Mater Sci. 2000;7:375-377.

28. Dannefaer S, Bretagnon T, Kerr D. Vacancy-type defects in crystalline and amorphous $\mathrm{SiO}_{2}$. J Appt Phys. 1993;7:884-890. doi:10.1063/1.354882

29. Dlubek G, Clarke AP, Fretwell HM, Dugdale SB, Alam MA. Positron lifetime studies of free volume hole size distribution in glassy polycarbonate and polystyrene. Phys Status Solidi A. 1996;157:351-364. doi:10.1002/pssa.2211570218

30. Dlubek G, Saarinen K, Fretwell HM. Positron states in polyethylene and polytetrafluoroethylene: A positron lifetime and Dopplerbroadening study. Nucl Instr Meth Phys Res B. 1998;142:139-155. doi:10.1016/S0168-583X(98)00261-4

31. Pfeifer CS, Shelton ZR, Braga RR, Windmoller D, Machalo JC, Stansbury JW. Characterization of dimethacrylate polymeric networks: A study of the crosslinked structure formed by monomers used in dental composites. Eur Polym J. 2011;47:162-170. doi:10.1016/j.eurpolymj.2010.11.007

32. Kluin JE, Yu Z, Vleeshouwers S, McGervey JD, Jamieson AM, Simha R. Temperature and time dependence of free volume in bisphenol A polycarbonates studied by positron lifetime spectroscopy. Macromolecules. 1992;25:5089-5093. doi:10.1021/ma00045a040

33. Kluin JE, YuZ, Vleeshouwers S, et al. Ortho-positronium lifetime studies of free volume in polycarbonates of different structures: Influence of hole size distribution. Macromolecules. 1993;26:1853-1861. doi:10.1021/ma00060a010

34. Ingram A. Atomic-deficient nanostructurization in water-sorption alumomagnesium spinel ceramics $\mathrm{MgAl}_{2} \mathrm{O}_{4}$. Appl Nanosci. 2019;9:731-735. doi:10.1007/s13204-018-0696-x 